\title{
Norois
}

Environnement, aménagement, société

$196 \mid 2005 / 3$

Géographie du littoral : approche géomatique

\section{La télédétection et les SIG dans les espaces côtiers. Eléments de synthèse à travers le parcours de François Cuq}

Progress in Remote Sensing and GIS technology used in coastal areas through the contribution of François $\mathrm{Cuq}$

Marc Robin et Françoise Gourmelon

\section{OpenEdition}

Journals

Édition électronique

URL : http://journals.openedition.org/norois/368

DOI : $10.4000 /$ norois.368

ISBN : 978-2-7535-1544-4

ISSN : 1760-8546

Éditeur

Presses universitaires de Rennes

Édition imprimée

Date de publication : 1 septembre 2005

Pagination : 11-21

ISBN : 978-2-7535-0218-5

ISSN : 0029-182X

Référence électronique

Marc Robin et Françoise Gourmelon, "La télédétection et les SIG dans les espaces côtiers. Eléments de synthèse à travers le parcours de François Cuq », Norois [En ligne], 196 | 2005/3, mis en ligne le 15 décembre 2008, consulté le 19 avril 2019. URL : http://journals.openedition.org/norois/368 ; DOI : 10.4000/norois.368

Ce document a été généré automatiquement le 19 avril 2019

(c) Tous droits réservés 


\title{
La télédétection et les SIG dans les espaces côtiers. Eléments de synthèse à travers le parcours de François Cuq
}

\author{
Progress in Remote Sensing and GIS technology used in coastal areas through \\ the contribution of François Cuq
}

Marc Robin et Françoise Gourmelon

\section{NOTE DE L'ÉDITEUR}

Cet article a été reçu le 26 avril 2005 et définitivement accepté le 26 octobre 2005.

\section{Un espace littoral complexe et vulnérable}

1 La zone côtière est un espace exigu, caractérisé par un linéaire considérable et par une pression humaine importante. Elle est le siège de multiples enjeux environnementaux, économiques et humains. Depuis quelques années, le littoral se «maritimise » de par une concentration croissante des usages en mer côtière (Gourmelon et Robin, 2005). De cette situation résultent des conflits d'usages et d'accès aux ressources, difficilement gérables $\mathrm{du}$ fait d'une approche sectorielle des problèmes et d'une juxtaposition de statuts et de compétences administratives et juridiques. Partout dans le monde, la zone côtière apparaît comme un espace vulnérable où les événements météo-marins se combinent à des pressions exercées par des activités humaines parfois conflictuelles, favorisant des risques d'origine naturelle et/ou anthropique.

Dans ce contexte, le besoin d'une approche plus dynamique et plus intégrée de la zone côtière, à travers l'interface terre-mer, se traduit depuis les années 1970 par le concept 
international de Gestion Intégrée de la Zone Côtière (GIZC) ${ }^{1}$ qui exprime le besoin d'agir collectivement sur les processus naturels et anthropiques susceptibles de menacer le maintien durable de la qualité de l'environnement et des activités (Cicin-Sain et Knecht, 1998). Le préalable à une gestion efficace de cet espace complexe implique une connaissance approfondie des processus qui s'y déroulent et une approche conceptuelle de son fonctionnement et de son évolution. Ce n'est qu'à partir des années 1980 que la communauté scientifique s'est intéressée aux zones côtières comme l'atteste la mise en place de programmes ${ }^{2}$ et de réseaux ${ }^{3}$ de recherche nationaux et internationaux. Ces initiatives ont conduit notamment à développer l'approche théorique de la gestion de la zone côtière (Clark, 1995) et à favoriser des recherches pluridisciplinaires qui se heurtent néanmoins à la complexité des processus en cause, à l'éparpillement et à l'hétérogénéité des compétences et des données dans un vaste champ pluridisciplinaire et dans de multiples institutions (Dronkers et De Vries, 1999). Ce constat a entraîné la mise en place récente d'observatoires ${ }^{4}$ et de réseaux d'observations ${ }^{5}$ qui ont pour vocation de regrouper et d'assurer la pérennité des données, des connaissances et des savoir-faire. Il a également contribué à la mise en œuvre des systèmes d'information géographique (SIG) côtiers. Toutefois, il a fallu une lente maturation pour en arriver à développer des outils théoriquement opérationnels qui sont encore aujourd'hui relativement mal diffusés auprès des gestionnaires de l'espace côtier.

Cette contribution propose de revisiter l'évolution technologique et conceptuelle dans la prise en compte des objets littoraux et des problèmes de gestion intégrée au cours de ces trente dernières années. Elle fait le point notamment sur la contribution des scientifiques français et de François Cuq et son équipe en particulier aux progrès réalisés tant en termes de connaissances de l'environnement côtier que de développements géomatiques originaux.

\section{Avant 1980 : le « tout télédétection »}

4 Le lancement du premier satellite d'observation de la terre ERTS1 (Landsat 1) en 1972 marque le début de l'approche spatialisée en milieu côtier. Les premières « spatio-cartes " montrent pour la première fois les objets géographiques côtiers de façon synoptique aussi bien en mode statique que cinématique (Verger, 1982). En France, des équipes de recherche pluridisciplinaires se structurent progressivement, à l'image du groupe FRALIT qui s'intéresse au littoral océanique français et plus spécifiquement au marais poitevin et à ses marges (FRALIT, 1977, 1981). Les travaux menés permettent de tester et de développer de nouvelles approches en matière de traitements d'images satellitales, de cerner plus précisément l'objet géographique extrait et d'aborder le problème des bibliothèques de signatures spectrales et texturales qui doivent permettre d'automatiser l'interprétation des images de télédétection (Pratt, 1978). Partout dans le monde, la télédétection satellitale, et particulièrement l'extraction d'objets géographiques par des méthodes de classification automatisée, deviennent un enjeu scientifique important dans le vaste champ des sciences de l'environnement (Harralick, 1979). C'est dans cette logique que s'inscrit la thèse de F. Cuq (1983) concernant le traitement géométrique et les classifications automatiques d'images Landsat MSS sur le secteur de la baie de Ronces/ Oléron. L'occupation du sol en zone côtière grossièrement extraite des images correspond à l'idée répandue que l'image doit pouvoir fournir l'ensemble de l'information nécessaire à la connaissance et à la gestion. À cette époque, les résultats sont souvent obtenus sans 
pré-traitements atmosphériques, pourtant nécessaires mais peu développés dans les logiciels commercialisés. Des objets littoraux sont toutefois cartographiés pour la première fois de façon instantanée et précise à la fois dans leurs dimensions spatiale et temporelle, qu'il s'agisse par exemple d'inondations en zone côtière (Verger, 1978), de lignes de rivages instantanées (Pestre et Verger, 1980) ou d'éléments bathymétriques (Lysenga, 1978). Des simulations sur les futurs capteurs embarqués à bord des platesformes satellitales sont effectuées (Cuq et al., 1982), des algorithmes sophistiqués sont proposés pour le traitement spectral (Joly et al., 1983) et pour les approches texturales (Quach, 1979) tandis que la notion de "pré-traitement d'images " permettant d'évaluer les contributions de l'atmosphère dans la qualité de l'information géographique à extraire, fait son apparition (Deschamps et al., 1981). Plusieurs travaux de recherche proposent des synthèses sur les apports et les limites de la télédétection (par exemple R. Regrain, 1980) qui apparait progressivement comme une source d'information parmi d'autres. La mise en commun d'informations géographiques de sources variées pour la description des objets géographiques devient une condition indispensable à la connaissance exhaustive et à la gestion des territoires.

\section{De 1980 à 1990 : l'émergence des SIG}

5 Parallèlement à la sophistication des outils de traitement d'images et à la spécialisation des capteurs embarqués, la décennie 1980-1990 est marquée par la montée en puissance des systèmes d'information géographique (SIG). Conçus au Canada dès les années 1970 (Tomlinson, 1967), les SIG se sont propagés rapidement dans le monde entier. Ils s'inscrivent dans un ensemble d'approches, de méthodes et d'outils en constante évolution (la géomatique) qui concerne à la fois les moyens d'acquisition de données numériques localisées (géodésie, photogrammétrie, télédétection), mais aussi les méthodes de gestion, d'analyse et de représentation de l'information géographique (SIG). Dans cet ensemble, les SIG représentent la synthèse des progrès réalisés dans le traitement numérique de l'information géographique, puisqu'ils permettent de replacer dans un cadre cohérent les données géoréférencées acquises par différentes méthodes géomatiques, de les analyser et de les cartographier (Denègre, 1992).

6 À cette époque, malgré des travaux novateurs sur les SIG préfigurant leur inéluctable montée en puissance (Hill et al., 1983), les études côtières restent encore très physiographiques et centrées sur les technologies spatiales. Des objets spécifiques sont étudiés à partir des données délivrées par des capteurs de plus en plus variés et selon des algorithmes éprouvés ou en développement, qu'il s'agisse de végétaux marins (Kerambrun, 1984), de pollution marine (Burkhalter et Meyer, 1984), des houles (Cuq, 1989), de l'estran et des petits fonds (Cuq, 1984a, 1993) ou le recours à des capteurs nouveaux tels que ceux du satellite américain Seasat qui au cours de ses huit mois de fonctionnement produira une masse d'informations exploitée sur toute la décennie (Cuq, $1984 \mathrm{~b}$; Gierloff-Emdem, 1989). Ce satellite est un précurseur en matière d'altimétrie radar et d'images radar à synthèse d'ouverture qui offre de nombreuses possibilités en zone côtière (Le Provost, 1982). Il sera suivi par ERS1, lancé en 1991. Les apports de l'analyse radar pour la description de divers processus côtiers (houles, morphologie sous-marine, description de l'estran) sont décrits notamment par P. Forget et al. (1995) et F. Cuq et al. (2002a). 
7 En parallèle aux progrès réalisés en télédétection, l'apport des SIG à la connaissance de l'environnement côtier commence réellement à se faire sentir au cours de cette décennie. Les lignes de rivage instantanées impliquent la mise en œuvre de traitements importants (Pestre et Verger, 1980). En les combinant au sein d'un SIG à un modèle de marée, elles peuvent conduire à la construction de modèles numériques d'estran (Cuq, 1984c). La combinaison de données hypso-bathymétriques extraites de documents cartographiques, de données granulométriques et du résultat de traitements d'image permet aussi de modéliser la répartition spatiale d'espèces en zone intertidale (Madec, 1987). L'idée de dériver d'images aériennes ou satellitales des variables topométriques préfigure alors le recours au SIG pour archiver et confronter l'information produite à d'autres sources de données. À cette époque, la demande sociale exige aussi des documents opérationnels qui ne peuvent être réalisés qu'avec des données issues de la télédétection (Denis et al., 1987). L'arrivée sur le marché des logiciels "géographiques» de type Arc Info va permettre l'intégration progressive de l'imagerie dans les SIG. Une mise au point complète est ainsi réalisée en matière de remise à jour de documents d'occupation du sol par la télédétection posant les bases solides de la complémentarité de la télédétection et des SIG (Audrain, 1989). Les nombreux appels à projet scientifique, dans le cadre de la validation des données satellitales, manifestent alors la volonté de démontrer l'utilité du recours à l'iconographie spatiale dans un processus d'acquisition, de structuration et d'exploitation thématique de l'information géographique, comme l'illustrent, par exemple, les divers PEPS (Programme d'Évaluation Préliminaire de Spot) lancés dans la décennie ${ }^{6}$.

\section{Entre 1990 et 2000 : des SIG à maturité}

En 1996, les résultats du programme Télédétection-SHS (Cuq, 1997), dans la continuité du Programme National de Télédétection Spatiale (PNTS), témoignent de la nécessaire synergie entre les SIG et la télédétection :

- les pixels de l'image ne sont plus considérés comme des mesures en tant que telles mais comme le moyen d'accès à une information caractéristique de l'espace étudié ;

- l'analyse concerne des objets géographiques, des textures, des structures et des formes d'organisation spatiale qui traduisent l'état d'interactions multiples et les réponses (actuelles ou passées) des sociétés aux changements du paysage ;

- le plus souvent, l'information extraite de l'image doit être replacée dans un contexte thématique plus vaste de façon à pouvoir être interprétée de façon satisfaisante.

Une modélisation conceptuelle destinée à fusionner les deux modes de représentation de l'information géographique, "raster » et "vecteur » est proposée (Cuq et Gourmelon, 1996). Basée sur des protocoles d'intégration des données extraites des images dans les systèmes d'information géographique, cette modélisation conceptuelle témoigne de la complémentarité des différentes sources pour une définition pertinente et une « consistance » des objets géographiques.

Dès le début des années 1990, on observe un accroissement du nombre d'études environnementales, notamment sur le littoral, utilisant la synergie SIG-télédétection (Robin, 1995). On peut citer à titre d'exemples l'utilisation de chroniques de photographies aériennes, d'images satellitales et de données vectorielles contenues dans des SIG pour étudier la cinématique des zones humides (Williams et Lyon, 1997), la croissance des couverts végétaux des littoraux dunaires (Kutiel et al., 2003), les 
changements d'occupation des sols des provinces côtières (Cuq et al., 1996, 2001), les processus cinématiques et dynamiques des estrans (Pennober, 2000), l'évolution des mangroves (Fromard et al., 2004). À une échelle spatiale plus petite, la mise en place d'observatoires de la qualité des eaux côtières est rendue possible grâce à l'archive ERS2 (660 images disponibles entre 1996 et 1999), nécessitant la conceptualisation de véritables systèmes d'information adaptés aux observatoires de l'environnement côtier (Gade et Alpers, 1999). La masse d'information satellitale ne cesse en effet de croître et l'enjeu devient progressivement de maîtriser cette complexité, d'où l'émergence de nouveaux algorithmes destinés à «fouiller » les données (data mining) et à classer les objets avec plus de finesse. Les réseaux de neurones se révèlent à cet égard de puissants outils pour identifier les objets géographiques à partir de diverses sources de données et leur application en zone côtière, espace complexe par excellence, améliore significativement l'identification et le suivi de processus tels que l'occupation et l'utilisation des sols (Mas et al., 2004).

11 Avec le développement des recherches pluridisciplinaires et la disponibilité croissante d'informations géographiques, notamment en milieu marin, les SIG côtiers se multiplient (Wright et Bartlett, 1999 ; Loubersac et Populus, 2000 ; Cuq et al., 2002b ; Bartlett et Smith, 2004 ; Gourmelon et Robin, 2005). Pourtant leur composante logicielle est encore peu adaptée à la résolution des problématiques côtières du fait de la non prise en compte du caractère flou des limites géographiques en mer et surtout des dynamiques spatiotemporelles qui caractérisent cet espace d'interfaces. De plus leur développement est limité par l'absence de données géographiques de référence (Le Berre et al., 2000).

\section{Les années 2000 : la promotion d'une information géographique de référence et la prise en compte de la dynamique par le couplage des SIG et des modèles}

Dès le début de cette décennie, l'absence d'informations géographiques de référence sur le littoral est apparue comme un frein à la réalisation des objectifs de la GIZC (Robin et al., 2005). Cette préoccupation internationale s'est traduite, en France, par de nombreuses initiatives telles que la réalisation de diverses bases d'information géographique par le $\mathrm{SHOM}^{7}$, du système BOSCO par le $\mathrm{CETMEF}^{8}$ et le $\mathrm{BRGM}^{9}$, du SIG interministériel littoral (SIGI), de l'Observatoire du littoral sous le pilotage de la DATAR. Elle s'est aussi traduite par la mise en place d'observatoires de l'environnement qui favorisent le regroupement des initiatives, des savoir-faire et des données. De plus, la stratégie nationale s'est concrétisée par les travaux du groupe de travail « littoral » (GTL) du CNIG ${ }^{10}$ qui rassemble les principales institutions productrices d'informations géographiques en zone côtière. De manière à proposer des données en adéquation avec les besoins des utilisateurs, le GTL a lancé une enquête qui confirme le besoin de la mise en place d'un Référentiel Géographique pour le littoral (RGL) constitué par des données continues terre-mer. Le récent projet Litto3D (SHOM/IGN) répond à ce besoin, et constitue la future composante altimétrique et bathymétrique du RGL. De plus le GTL a réalisé un inventaire des données géographiques disponibles sur l'ensemble des littoraux français. Dans le contexte du développement des connaissances et de la GIZC, les conditions d'accessibilité aux données apparaissent comme un élément essentiel. Il en émane des recommandations internationales visant à une certaine rationalisation du transfert des données du producteur à l'utilisateur. Les initiatives de catalogage selon des normes cohérentes et la 
création de portails Web pour le porter à connaissance sont encouragées à tous les niveaux locaux, régionaux, nationaux et internationaux.

En plus du développement des SIG et de la multiplication des données satellitales obligeant à réfléchir en termes d'observatoire, apparaît la nécessité d'intégrer la dimension temporelle dans les SIG et la dimension spatiale dans les plates-formes de modélisation de façon à prendre en compte la dynamique des systèmes (Van Zuidam et al., 1998). En milieu côtier, il apparaît que la mesure de l'impact des activités humaines constitue à la fois l'un des principaux enjeux de la gestion intégrée des zones côtières et la difficulté majeure que rencontre l'étude pluridisciplinaire de la zone littorale depuis une vingtaine d'années (Cuq, 2000). Cette difficulté est liée à plusieurs causes : la complexité de l'analyse du rôle des sociétés humaines en raison de leur caractère non prévisible à long terme, la méfiance des chercheurs en sciences sociales vis-à-vis des approches déterministes et l'absence d'informations structurées décrivant l'impact de l'homme sur le milieu. Dans ce contexte, la compréhension des interactions nature-société, à l'interface terre-mer amène les scientifiques à œuvrer dans un cadre pluridisciplinaire afin de répondre à cette exigence de spatialisation des processus dynamiques en zone côtière.

Les systèmes d'information géographique sont, pour la plupart, élaborés pour étudier des phénomènes statiques ou dynamiques selon une approche qui reste statique même si une succession d'états selon un pas de temps donné peut être prise en compte. Le couplage des SIG avec des modèles permet l'accès à la dynamique des processus et le SIG devient source et réceptacle de ce que produit le modèle, en procurant non seulement les données de calibration mais aussi les données de validation (Pouliot, 1998). Avant les années 1980, les développements des SIG et des modèles, notamment en hydrologie, sont parallèles ; aucune passerelle n'étant envisagée pour des raisons principalement techniques (Sui et Maggio, 1999). Dès 1990, des solutions logicielles de couplage font leur apparition: par exemple le package RIBS (Real-time Interactive Basin Simulator) intégrant un modèle de prédiction des précipitations reposant sur des informations radar, un modèle de ruissellement basé sur un modèle numérique de terrain, et diverses informations relatives au bassin versant destinées à la prise en compte du temps réel dans le ruissellement et les inondations (Garrote et Bras, 1995). Des interfaces entre SIG et modèles de simulation sont développées afin de doter les SIG d'un accès au temps réel et à la dynamique des processus. C'est par exemple le cas de la plate-forme GRASS interfacée à des outils de simulations (WEPP, Water Erosion Prediction Project) (Engel et al., 1993) et du couplage d'Arc Info avec le système HMS (Hydrologic Modeling System). Mais c'est plus récemment que des solutions de couplage dans l'environnement Windows ont permis l'essor de plates-formes accessibles au plus grand nombre: c'est par exemple le cas du couplage d'ArcView GIS avec le modèle SWAT (Soil land Water Assessment Tool) (Luzio et al., 2002) ou encore des modules développés par compartiments hydrologiques depuis les bassins versants jusqu'à la mer côtière par DHI Water \& Environment. La tendance actuelle est, au-delà des couplages, d'avoir recours aux ressources d'Internet pour l'accès aux bases de données distribuées, décuplant les possibilités d'accès au temps réel (AlSabhan et al., 2003).

Dans le cadre des recherches visant à décrire les transferts de polluants entre les domaines terrestres et marins (bassins versants-mer côtière), l'intérêt d'un couplage SIGmodèle est triple. Il permet de comprendre le fonctionnement d'un bassin versant et les relations qui existent entre les pressions agricoles, le milieu et la qualité des eaux, en 
hiérarchisant le rôle des différentes variables. Ensuite, le couplage permet de cartographier les niveaux de pollution sur des secteurs non mesurés et d'évaluer les facteurs de risque et de vulnérabilité tels que la production d'azote, le stockage d'azote dans le sol ou dans la nappe aquifère, l'intensité du ruissellement ou encore les variations de l'humidité et des niveaux d'eau dans des zones humides (Zacharias et al., 2005). Enfin, le couplage SIG-modèle peut favoriser la prédiction en étudiant l'impact des changements d'utilisations des sols et d'activités humaines sur la qualité des eaux. On peut ajouter à ces trois éléments la possibilité d'offrir à l'exutoire des bassins versants côtiers les valeurs de pollution qui peuvent être reprises dans un modèle hydrodynamique pour simuler le suivi et l'impact de la pollution venant du bassin versant vers la mer côtière (Loubersac et al., 2000).

Diverses études formalisent et appliquent ces couplages pour répondre à des problématiques environnementales. Par exemple, la simulation de l'activité d'élevage intensif en zone côtière a été réalisée à l'aide d'une approche multi-agent pour évaluer, à travers la modélisation des épandages, la potentialité de pollution dans le bassin versant (Tissot et Cuq, 2004 ; Tissot et al., 2004, sous presse). On se place ici à l'amont d'un modèle de transfert et la simulation concerne l'activité (élevage porcin intensif) qui amène dans les espaces communaux selon leurs spécificités environnementales et le filtrage réglementaire une quantité de polluant mobilisable. Un modèle de transfert de polluant de type semi-distribué peut aussi être utilisé afin de suivre sa trajectoire à travers le bassin versant jusqu'à l'exutoire (Lomakine, 2005). Sur d'autres problématiques côtières, le couplage SIG - plate-forme de simulation peut être utilisé pour mettre en évidence des conflits potentiels entre activités concurrentielles et leur impact sur la ressource en fonction des contraintes (réglementaires, environnementales, météorologiques...) régissant leur déroulement dans le temps et dans l'espace (Le Tixerant, 2004).

\section{Conclusion : SIG et sphères d'acteurs}

17 Si des progrès importants ont été réalisés au cours de ces trois dernières décennies dans le traitement de l'information géographique et dans la diffusion des résultats de la recherche vers la société civile, il n'empêche que le caractère opérationnel d'un SIG dépend de la décision de partager les savoir-faire et les informations à tous les niveaux de la société et de se doter des moyens permettant d'y accéder. Les facettes politiques, sociales et individuelles de l'introduction des technologies géospatiales dans l'aide à la décision sont primordiales (Caron, 2000). Certains modèles géomatiques prônent la mise en place d'infrastructures dont l'intérêt actuel, dans la société de l'information est discutable. D'autres s'intéressent à l'intégration des SIG dans des systèmes d'information conçus pour rendre facilement accessibles les produits de la recherche (Guarnieri et al., 2003). Dans ce cas, la composante organisationnelle des SIG est négligée au profit du transfert des données et des compétences. Si les SIG alimentés par la télédétection et couplés à des plates-formes de simulation sont actuellement utilisés dans de nombreuses problématiques littorales, scientifiques et finalisées, leur appui à la prise de décision dans un contexte de gestion intégrée de la zone côtière reste actuellement partiel et dans bien des cas sectoriel. Néanmoins, sur le thème du risque côtier, ils fournissent un certain nombre d'éléments utiles à l'évaluation et à la simulation du risque : cartes thématiques, production d'indices et de variables spatio-temporelles synthétiques, représentation globale du risque (Robin, 2002). Si l'on admet qu'une décision de gestion a pour objectif de 
maintenir ou d'améliorer l'état du système dans des perspectives de développement durable, il est indispensable d'élaborer un modèle de réalité adéquat. Or la complexité des systèmes côtiers et des processus qui s'y déroulent rendent difficile sa conception et impliquent d'étudier les modalités d'intégration des SIG au sein de systèmes d'aide à la décision fondés sur la complémentarité des méthodes géomatiques (télédétection, photogrammétrie, géodésie spatiale, SIG) et notamment sur le couplage des SIG avec des modèles (Capobianco, 1999). La compréhension des interactions entre nature et sociétés représente l'un des enjeux de la gestion intégrée des zones côtières, au niveau de la simulation de l'impact et de la prédiction des changements (Cuq, 2000).

L'exercice rétrospectif est riche d'enseignements sur l'évolution de la demande sociale en relation avec les possibilités technologiques du moment. On pourrait se livrer à un exercice de prospective en la matière à la lueur d'expériences «SIG » menées depuis de nombreuses années sur diverses zones côtières dans un contexte de préservation et de développement durable (Gourmelon et Le Berre, 2004 ; Gourmelon et al., sous presse). Le constat d'une appropriation insuffisante par les acteurs en charge de la gestion du littoral est toujours d'actualité. Il apparaît donc que l'enjeu actuel est d'associer systématiquement les acteurs locaux à la mise en place des SIG de façon à leur conférer un espoir de pérennité, fondement même de la notion d'observatoire, et de pallier ainsi la difficulté du dialogue entre décideurs, gestionnaires et scientifiques. Plus que jamais, le travail doit se faire au niveau des interfaces de la sphère scientifique proposant des outils, des données et des résultats, de la sphère de gestion potentiellement utilisatrice des résultats de la recherche et de la sphère de décision étant à l'amont de la demande sociale et devant accompagner l'émergence des propositions de la sphère scientifique.

\section{BIBLIOGRAPHIE}

AL-SABHAN (W.), MULLIGAN (M.), BLACKBURN (G. A.), 2003. - « A real-time hydrological model for flood prediction using GIS and the WWW », Computers,Environment and Urban System, $\mathrm{n}^{\circ} 27$, p. 9-32.

AUDRAIN (V.), 1989. - Méthodologie d'actualisation de documents cartographiques thématiques par télédétection aérienne et satellitaire, doctorat de Géographie, Université de Paris 1, 283 p.

BARTLETT (D. J.), SMITH (J.), 2004. - GIS for coastal zone management, Londres, CRC Press, 310 p.

BURKHALTER (R.), MEYER (C.), 1984. - « The operational oil pollution surveillance system being used in France », dans Remote sensing for the control of marine pollution, Plenum Press, p. 87-100.

CAPOBIANCO (M.), 1999. - « On the integrated modelling of coastal changes », Journal of Coastal Conservation, $\mathrm{n}^{\circ}$ 5, p. 113-124.

CARON (C.), 2000. - « Les projets de géomatisation : contexte social et organisationnel », Revue Internationale de Géomatique, vol. 10, n² 2/2000, p. 167-192.

CICIN-SAIN (B.), KNECHT (R.), 1998. - Integrated coastal and ocean management, Washington, Island Press, $517 \mathrm{p}$.

CLARK (J. R.), 1995. - Coastal zone management Handbook, Boca Raton, Lewis publishers, 694 p. 
CUQ (F.), 1983. - Télédétection du littoral saintongeais : méthodes de traitement et interprétation d'images satellitaires, doctorat de Géographie, Montrouge, collection ENSJF, n² 23, 192 p.

-, 1984a. - «Exemples de cartographie de phénomènes littoraux », L'Espace géographique, $\mathrm{n}^{\circ}$ 3, p. 265-271.

-, 1984b. - « Interprétation automatique de champs de vagues à partir des données du radar SAR de Seasat ", Photo-Interprétation, $\mathrm{n}^{\circ} 84-4$, fascicule 1, $4 \mathrm{p}$.

-, 1984c. - « Constitution d'un modèle hypsométrique par analyse diachronique de trois simulations Spot ", dans Actes eighteenth International Symposium on Remote Sensing of Environment, Paris, p. 1955-1960.

-, 1989. - « Mise en évidence de la houle à la côte à partir d'images Spot en modes multibande et panchromatique ", Revue française de Photogrammétrie et de Télédétection, n 114, p. 28-30.

-, 1993. - « Remote sensing of sea-surface and coastal features in the area of the Golfe d'Arguin, Mauritania », Hydrobiologia, n² 258, p. 33-40.

-, 1997. - Journées de la télédétection en sciences de l'homme et de la société, Photo-Interprétation , nº spécial, vol. 35, 144 p.

-, 2000. - « Systèmes d'information géographique et gestion intégrée des zones côtières ", dans L OUBERSAC (L.), Populus (J.) (dir.), Coastgis'99: Geomatics and coastal environment, Brest, éditions Ifremer, p. 18-29.

CUQ (F.), GOURMELon (F.), 1996. - « Conditions d'intégration des données satellitaires au sein de bases d'information géographique », Revue internationale de Géomatique, n 4, p. 349-363.

CUQ (F.), BONNAFOUX (G.), BLERARD (C.), 2002a. - « Analyse radar de l'environnement littoral », dans B ARON-YELLÈs (N.), GOELDNER-GIANELLA (L.), VELUT (S.) (dir.), Le littoral : regards, pratiques et savoirs; études offertes à Fernand Verger, Paris, édition Rue-d'Ulm, p. 117-134.

CUQ (F.), DEVOGÈLE (T.), POPULUS (J.), 2002b. - SIG côtiers, $\mathrm{n}^{\circ}$ spécial de la Revue internationale de Géomatique, vol. 12, n³/2002, 389 p.

CUQ (F.), CAMPREDon (P.), GiRAUdet (J.), GIRAUDET (E.), GOURMELON (F.), PENNOBER (G.), DA SILVA (A. S.), 2001. - Un Système d'Information Géographique pour l'aide à la gestion intégrée de l'archipel des Bijagos (GuinéeBissau), CNRS/UICN, Carte (feuilles à 1/50 000) et notice, 88 p.

CUQ (F.), MADEC (V.), THOMAS (Y.-F.), ZBINDEN (R.), 1982. - Étude des données simulées du satellite Spot sur le goulet de Fromentine et ses abords, collection système Spot d'observation de la Terre, Association québécoise de Télédétection et Société Française de Photogrammétrie et Télédétection, Montréal, p. 239-257.

CUQ (F.), MADEC (V.), GOURMELON (F.), 1996. - « Mise à jour de la carte d'occupation des sols des provinces côtières de Guinée-Bissau », Mappemonde, $\mathrm{n}^{\circ}$ 4, p. 21-26.

DENÈGRE (J.), 1992. - « Nouvelles technologies au service de l'investigation géographique : le rôle de la télédétection spatiale et des systèmes d'information géographique ", SIGAS, vol. 2, $\mathrm{n}^{\circ} 2, \mathrm{p}$. 139-149.

DENIS (J.), GROTTE (A.), POPULUS (J.), 1987. - Application de l'imagerie Spot à la préparation de plans d'intervention contre les pollutions par hydrocarbures, Spot 1, utilisation des images, bilan résultats, dans rapport CNES, p. 1069-1078. 
DESCHAMPS (P.-Y.), HERMAN (M.), TANRÉ (D.), 1981. - « Influence de l'atmosphère en télédétection des ressources terrestres. Modélisation et possibilité de correction », dans actes du $1^{\mathrm{er}}$ colloque international Signatures spectrales d'objets en télédétection, Avignon, INRA, p. 543-558.

DRONKERS (J.), DE VRIES (I.), 1999. - « Integrated coastal management : the challenge of transdisciplinarity ", Journal of Coastal Conservation, $n^{\circ}$ 5, p. 97-102.

ENGEL (B. A.), SRINIVASAN (R.), ARNOLD (J.), REWERTS (C.), BROWN (S. J.), 1993. - « Nonpoint source (NPS) pollution modeling using models integrated with geographic information systems (GIS) », Water Science and Technology, $\mathrm{n}^{\circ} 28$ (3-5), p. 685-690.

FORGET (P.), BROCHE (P.), CUQ (F.), 1995. - « Principles of swell measurements by SAR with application to ERS1 observations off the Mauritanian coast », International Journal of Remote Sensing, vol. $16, \mathrm{n}^{\circ}$ 13, p. 2403-2422.

FRALIT, 1977. - Télédétection du littoral océanique de la France, Montrouge, collection ENSJF, $\mathrm{n}^{\circ} 11$, $312 \mathrm{p}$.

-, 1981. - Télédétection du marais poitevin et de ses marges, Montrouge, collection ENSJF, nº 13, 312 p.

FROMARD (F.), VEGA (C.), PROISY (C.), 2004. - « Half a century of dynamic coastal change affecting mangrove shorelines of French Guiana. A case study based on remote sensing data analyses and field surveys ", Marine Geology, vol. 208, n 2-4, p. 265-280.

GADE (M.), ALPERS (W.), 1999. - « Using ERS-2 SAR images for routine observation of marine pollution in European coastal waters ", The Science of The Total Environment, vol. 237-238, p. 441-448.

GARROTE (L.), BRAS (R. L.), 1995. - « An integrated software environment for real-time use of a distributed hydrologic model », Journal of Hydrology, n 167, p. 307-326.

GIERLOFF-EMDEM (H. G.), 1989. - « Remote Sensing with the Seasat altimeter », dans Actes $2^{e}$ conférence internationale de Géomorphologie, GSTS-SERTIT, p. 66-79.

GOURMELON (F.), LE BERRE (I.), 2004. - « Towards an institutional GIS for the Iroise Sea (France) », dans BARTLETT (D.), SMITH (J.) (dir.), GIS for Coastal Zone Management, New York, CRC Press, p. 147-152.

GOURMELON (F.), ROBIN (M.), 2005. - SIG et littoral, Paris, éditions Hermès, traité IGAT, 328 p. GOURMELON (F.), AFFiAn (K.), GEORIS CRESEVEAu (J.), PENNOBER (G.), ROBIN (M.), (sous presse). - « Apports des SIG à la connaissance et à la gestion de l'environnement côtier, exemples choisis en Mauritanie, Guinée-Bissau et Côte-d'Ivoire ", dans actes du symposium international Ecosystèmes côtiers d'Afrique de l'Ouest, Bruxelles.

GUARNIERI (F.), GARBOLINO (E.), HOULLIER (F.), CUQ (F.), LÉVÊQUE (C.), WEILl (A.), MATARASSO (P.), 2003. -

«Contribution à la définition opérationnelle et à la modélisation de la mémoire

environnementale », dans Quelles natures voulons-nous ?, Paris, Elsevier, p. 296-307.

HARRALICK (R. M.), 1979.- « Statistical and structural approaches to texture », in proceedingIEEE, $\mathrm{n}^{\circ}$ 67, p. 786-804.

HILL (J. M.), HARLOW (C. A.), ZIMMERMAN (P.), 1983. - « Geographic information systems as applied to the manipulation of environmental data ", The Environmentalist, vol. 3, n 1, p. 33-38. 
JOLY (G.), VERGER (F.), CRETTEZ (J.-P.), 1983. - « Classification des données Landsat unitemporelles et multitemporelles par la transformation de Karhunen-Loeve (application à l'anse de l'Aiguillon) », L'Espace Géographique, p. 125-141.

KERAMBRUN (L.), 1984. - Contribution à l'étude de la fertilité des fonds rocheux côtiers de Bretagne, doctorat de Géographie, Université de Bretagne Occidentale, 151 p.

KUTIEL (P.), COHEN (О.), SHOSHANY (M.), SHUB (M.), 2003. - « Vegetation establishment on the southern Israeli coastal sand dunes between the years 1965 and 1999 », Landscape and Urban Planning, vol. 67, n' 1-4, p. 141-156.

LE BERRE (I.), MEYRAT (J.), PASTOL (Y.), 2000 - - «Application des données hydrographiques à l'étude synthétique de l'environnement côtier : exemple d'un SIG sur le littoral du Finistère (France) », dans Populus (J.), LOUBERSAC (L.) (coord.), CoastGIS'99: Geomatics and coastal environment, Plouzané, éditions Ifremer, p. 233-244.

LE PROVOST (C.), 1982. - « Mesure altimétrique par satellite de la topographie de la surface libre de la mer en zone littorale ", L'océanologie spatiale, p. 619-642.

LE TIXERANT (M.), 2004. - Dynamique des activités humaines en mer côtière : application à la mer d'Iroise, doctorat de Géographie, Université de Bretagne Occidentale, 195 p.

LOMAKINE (C.), 2005. - Contribution des bassins versants aux pollutions des écosystèmes conchylicoles du Croisic et de Pen-Bé, approche par couplage d'un SIG avec un modèle agro-hydrologique, doctorat de Géographie, Université de Nantes, 308 p.

LoubersaC (L.), SALOMON (J.-C.), BRETON (M.), DURAND (C.), GAUdineAu (C.), 2000. - « Perspectives offertes par la communication entre un modèle hydrodynamique et un SIG pour l'aide au diagnostic environnemental : caractérisation de la dynamique et la qualité des masses d'eaux côtières ", dans Populus (J.), LOUBERSAC (L.) (coord.), CoastGIS'99: Geomatics and coastal environment, Plouzané, éditions Ifremer, p. 173-185.

LOUBERSAC (L.), POPULUS (J.), 2000. - Coastgis'99 : Geomatics and coastal environment, Plouzané, éditions Ifremer, $318 \mathrm{p}$.

LUZIO (D. M.), SRINIVASAN (R.), ARNOLD (J. G.), 2002. - « Integration of watershed tools and SWAT model into BASINS ", Journal of The American Water Resources Association, $n^{\circ} 38$ (4), p. 1127-1141.

LYSENGA (D. R.), 1978. - « Passive remote sensing techniques for mapping water depth and bottom features », Applied Optics, n 13, p. 379-383.

MADEC (V.), 1987. - Méthodes de constitution et d'exploitation d'une base de données images sur la baie du Mont Saint-Michel, doctorat de Géographie, Université de Bretagne Occidentale, 169 p.

MAS (J. F.) et al., 2004. - « Mapping land use/cover in a tropical coastal area using satellite sensor data, GIS and artificial neural networks ", Estuarine, Coastal and Shelf Science, vol. 59, $\mathrm{n}^{\circ}$ 2, p. 219-230.

PESTRE (C.), VERGER (F.), 1980. - « Géomorphologie de la baie du Mont-Saint-Michel par télédétection des lignes de rivage instantanées ", dans Actes $3^{e}$ colloque international du GDTA, Toulouse, p. 175-181.

PENNOBER (G.), 2000. - «Typologie dynamique de la zone intertidale de l'archipel des Bijagos (Guinée-Bissau) », dans PoPUlus (J.), LOUBERSAC (L.) (coord.), CoastGIS'99 : Geomatics and coastal environment, Plouzané, éditions Ifremer, p. 255-266. 
POULIOT (J.), 1998. - « Définition d'un référentiel pour le couplage SIG-modèle ", dans Actes $4^{e}$ journées scientifiques du département de génie rural, Montana (Suisse), PFL, [http:// sirs.scg.ulaval.ca/jacynthe/doctorat/article_JS_98.html].

PRATT (W. K.), 1978. - Digital Image Processing, Willey Inter-Sciences.

QUACH (J.), 1979. - Utilisation de transformations locales pour l'étude de la texture des images de télédétection, doctorat de Géographie, Université Paul Sabatier (Toulouse), 120 p.

REGRAIN (R.), 1980. - Géographie physique et télédétection des marais charentais, Abbeville, Paillard, $512 \mathrm{p}$.

ROBIN (M.), 1995. - La télédétection : des satellites aux SIG, Paris, Nathan, $320 \mathrm{p}$

-, 2002. - «Étude des risques côtiers sous l'angle de la géomatique », Annales de Géographie, n 627-628, p. 471-502.

ROBIN (M.), MORSETTI (V.), PICHEREAU (D.), BABILLOT (P.), COLAS (S.), TEXIER (C.), THUILLIER (A.), 2005. "L'information géographique de référence en zone côtière : la situation française ", dans $G$ ourmelon (F.), Robin (M.) (dir.), SIG et littoral, Paris, Hermès, traité IGAT, p. 49-83.

SUI (D. Z.), MAGGIO (R. C.), 1999. - « Integrating GIS with hydrological modeling : practices, problems and prospects, Computers ", Environment and Urban Systems, $\mathrm{n}^{\circ} 23, \mathrm{p} .33-51$.

TISSOT (C.), CUQ (F.), 2004. - « Apport des SIG pour la modélisation spatio-temporelle d'activités humaines ", Revue Internationale de Géomatique, n 14 (1/2004), p. 83-96.

TISSOT (C.), GOURMELON (F.), CUQ (F.), (sous presse). - « Modélisation d'une activité agricole à fort impact environnemental en zone côtière ", L'Espace Géographique.

TISSOT (C.), LE TIXERANT (M.), GOURMELON (F.), LE BERRE (I.), 2004. - « Modeling interactions between human activities and coastal zone environment », dans actes de la conférence internationale Littoral 2004 Delivering sustainable coasts: connecting science and policy, EUCC/Eurocoast, Aberdeen, Cambridge publications, p. 425-431.

TOMLINSON (R. F.), 1967. - An introduction to the Geographic Information System of the Canada Land Inventory, Department of Forestry and Rural Development, Ottawa.

VAN ZUIDAM (R. A.), FARIFTEH (J.), ELEVELD (M. A.), CHENG (T.), 1998. - « Developments in remote sensing, dynamic modelling and GIS applications for integrated coastal zone management », Journal of Coastal Conservation, $\mathrm{n}^{\circ} 4$, p. 191-202.

VERGER (F.), 1978. - «L'inondation du marais de Monts en Vendée d'après les données Landsat 2 du 13 décembre 1976 », Photo-interprétation, n 3, 3 p.

-, 1982. - L'observation de la terre par les satellites, Paris, PUF, coll. «Que sais-je ? », 128 p.

WILLIAMS (D. C.), LYON (J. G.), 1997. - « Historical aerial photographs and a geographic information system (GIS) to determine effects of long-term water level fluctuations on wetlands along the St. Marys River, Michigan, USA », Aquatic Botany, vol. 58, n 3-4, p. 363-378.

ZACHARIAS (I.), DIMITRIOU (E.), KOUSSOURIS (T.), 2005. - « Integrated water management scenarios for wetland protection : application in Trichonis Lake », Environmental Modelling and Software, $\mathrm{n}^{\circ}$ 20, p. 177-185.

WHRIGHT (D. J.), BARTLETT (D. J.), 1999. - Marine and coastal Geographical Information System, Londres, Taylor \& Francis, 320 p. 


\section{NOTES}

1. La première action gouvernementale en faveur de la GIZC est américaine et date de 1972. Le Coastal Zone Management Act a été mis en œuvre par le National Coastal Zone Management dans le cadre d'un partenariat volontaire entre le gouvernement fédéral et les Etats nord-américains. En Europe, la Stratégie européenne d'Aménagement Intégré (AIZC) ou Integrated Coastal Zone Management (ICZM) a été proposée à la fin des années 1990 par l'Union européenne. Elle est fondée sur un processus dynamique, continu et itératif destiné à promouvoir la gestion durable des zones côtières qui s'appuie sur la sensibilisation, la concertation et la coopération entre les acteurs. Sa mise en œuvre opérationnelle, conditionnée par un réseau de concertation complexe entre les institutions européennes et les administrations nationales, régionales et locales tarde toutefois à se concrétiser par des options politiques claires.

2. On peut citer le programme international LOICZ (Land-Ocean Interactions in the Coastal Zones) mis en place dans les années 1990 et sa contribution européenne ELOISE (European LandOcean Interaction Studies). En France, les programmes LITEAU et le PNEC (Programme National d'Environnement Côtier) se complètent depuis la fin des années 1990.

3. Par exemple, les réseaux de recherche associatifs européens Eurocoast (European Coastal Association for Science and Technology) et EUCC (European Union of Coastal Conservation) s'appuient sur des réseaux de chercheurs, d'ingénieurs et de gestionnaires. Ils ont pour objectif de diffuser les connaissances scientifiques et techniques concernant la protection et l'aménagement du littoral tout en assurant la promotion des approches pluridisciplinaires. Leur fonctionnement repose essentiellement sur l'organisation de colloques internationaux et sur l'édition de revues.

4. Aux États-Unis, le programme Long Term Environmental Research (LTER) s'appuie sur 24 structures opérationnelles. En France, certains Observatoires des Sciences de l'Univers (OSU) concernent la zone côtière.

5. Par exemple, l'Ifremer assure le fonctionnement de divers réseaux d'observations tels que REBENT (Réseau Benthique), RNO (Réseau National d'observation de la qualité du milieu marin), REMI (REseau de surveillance MIcrobiologique) et le REPHY (REseau de surveillance du PHYtoplancton).

6. PEPS $n^{\circ} 116,1987$, programme SPOLIT : Apport de Spot à l'étude de la physiographie et de l'aménagement du littoral vendéen et charentais, sous la responsabilité scientifique de F. Verger (IMAGEO-CNRS) et de P. Rebillard (Société Européenne de Propulsion, Division Traitements d'Images de Puteaux), M. Robin et G. Decroix (coll.).

7. Service Hydrographique et Océanographique de la Marine.

8. Centre d'Étude Technique Maritime et Fluviale.

9. Bureau de Recherches Géologiques et Minières.

10. Conseil National de l'Information Géographique.

\section{RÉSUMÉS}

La géographie a été renouvelée depuis une trentaine d'années par l'émergence de l'informatique qui a permis tour à tour de voir arriver à maturité la télédétection, puis l'intégration des données 
satellitales au sein de systèmes d'information géographique en cours de structuration, l'arrivée à maturité des SIG et enfin en dernier lieu le couplage entre les SIG et les modèles afin d'insérer la dynamique des processus dans la structuration et la représentation des objets géographiques. Le littoral, la connaissance de son fonctionnement et sa gestion intégrée, ont bénéficié de cette avancée technologique et conceptuelle. Cet article se propose de dresser la synthèse des progrès réalisés depuis les années 1970 par la communauté géographique, notamment à travers les travaux de François Cuq.

Since 1970's, environmental sciences, in particular Geography, have been known crucial evolutions due to computer science. Remote sensing and GIS technologies, integration of image classification into databases, and actually the coupling of GIS with numeric models have contributed to the "renouveau" of the geographical approach and have provided opportunities for environmental studies dealing with the coastal zones. This contribution aims to present the improvments due to the geographers (and especially François Cuq) since 1970's.

\section{INDEX}

Mots-clés : géomatique, SIG, gestion intégrée des zones côtières

Keywords : geomatic, integrated coastal zone management, GIS, remote sensing

\section{AUTEURS}

\section{MARC ROBIN}

Géolittomer - UMR 6554 CNRS LETG, Université de Nantes, Chemin de la Censive du Tertre, BP 81307, 44313 Nantes cedex 3, France, marc.robin@univ-nantes.fr

\section{FRANÇOISE GOURMELON}

Géomer - UMR 6554 CNRS LETG, Université de Bretagne Occidentale, IUEM, Technopôle BrestIroise, 29280 Plouzané, France,

Francoise.Gourmelon@univ-brest.fr 